\title{
POLYMERIC NANOPARTICLES FOR IMPROVED BIOAVAILABILITY OF CILNIDIPINE
}

\author{
ROHIT MISHRA', SHOWKAT R MIR², SAIMA AMIN ${ }^{1 *}$
}

1Department of Pharmaceutics, Faculty of Pharmacy, Jamia Hamdard, New Delhi 110062, ${ }^{2}$ Phytopharmaceutical Laboratory, Department of Pharmacognosy and Phytochemistry, Faculty of Pharmacy, Jamia Hamdard, New Delhi 110062 Email: samin@jamiahamdard.ac.in

Received: 20 Oct 2016 Revised and Accepted: 27 Feb 2017

\section{ABSTRACT}

Objective: In the present study, we aimed to optimize, characterize and evaluate poly lactic-glycolic acid nanoparticles of cilnidipine for improved permeation across the gastrointestinal tract.

Methods: Poly lactic-glycolic acid-cilnidipine (PLGA-CIL) nanoparticles were prepared by an emulsification solvent evaporation/diffusion method using polyvinyl alcohol (PVA) as a surfactant. The prepared nanoparticles were successfully characterized for particle size, shape, drug release and pharmacological effect.

Results: Polymeric nanoparticles of cilnidipine at a dose of $10 \mathrm{mg}$ had a small particle size of $272 \mathrm{~nm}$ with smooth morphology. Nearly $81 \%$ of the drug was encapsulated in the polymeric structure and showed $18.99 \pm 0.59 \%$ of release at $\mathrm{pH} 1.2$ within $3 \mathrm{~h}$, however, at $\mathrm{pH} 6.8$ the release was $80.89 \pm 1.59 \%$. The formulation had a better antihypertensive effect on methylprednisolone-induced hypertensive rats. The relative bioavailability of the nanoparticles was found to be 2.44 and 2.94 fold higher than the tablet and drug suspension respectively.

Conclusion: The results demonstrated that the novel delivery system offers an effective strategy for treatment of hypertension.

Keywords: Polymeric nanoparticles, Bioavailability, Solvent evaporation, Polymers

(C) 2017 The Authors. Published by Innovare Academic Sciences Pvt Ltd. This is an open access article under the CC BY license (http://creativecommons.org/licenses/by/4. 0/A DOI: http://dx.doi.org/10.22159/ijpps.2017v9i4.15786

\section{INTRODUCTION}

Cilnidipine chemically known as 1,4-dihydro-2,6-dimethyl-4-(3nitrophenyl)-3,5-pyridinedicarboxylic acid 2-methoxyethyl (2E)-3phenyl-2-propenyl ester, is the fourth generation calcium channel blocker used in the treatment of hypertension. It inhibits cellular influx of calcium thus causing vasodilatation. Amongst all antihypertensive drugs, it only exerts renoprotective, neuroprotective and cardioprotective action. It is a yellow, odourless crystalline powder with molecular weight $492 \mathrm{~g} / \mathrm{mol}$. As per Biopharmaceutical Classification System (BCS), it belongs to class II drug as has low aqueous solubility and high permeability. Due to low aqueous solubility, it has poor dissolution leading to low oral bioavailability from an effective dose of $10 \mathrm{mg} \mathrm{p}$. o. necessitating dose escalation. Oral administration also brings gastrointestinal side effects like pain, nausea, constipation and gastritis [1]. A few attempts to improve the solubility of cilnidipine like inclusion complex of cilnidipine with $\beta$-cyclodextrin, coevaporation with polymers, solid dispersions using different carriers have been reported in the literature [2]. But none of these substantiated the improved bioavailability.

In recent trends, polymeric nanoparticles also known as ultrafine particles sized between 10-100 nanometre are attractive for medical purposes as they are able to bind, absorb and carry drugs and proteins [3]. In nanoparticles, the drug is dissolved, attached, encapsulated or entrapped to a nanoparticle matrix or carrier. The principle reason in designing a nanoparticle is to optimise the size, the surface properties and to control the release of the drug for complete absorption [4]. Biocompatible and biodegradable polymers such as polylactic acid (PLA), polyglycolic acid (PGA), poly lactic-glycolic acid (PLGA) have attracted considerable attention as potential drug delivery devices as the Food and Drug Administration (FDA) has approved them for micro and nanoparticles and for a number of therapeutic devices such as grafts, sutures, implants and prosthetic devices [5]. Literature supports that PLGA nanoparticles are known to protect poorly soluble and unstable payloads from the biological milieu and are small enough for capillary penetrations; cellular internalization and endosomal escape [6]. Therefore, in the present study, an attempt was made to develop and characterise an optimised polymeric nanoparticle using PLGA (75:25) as a polymer and PVA (polyvinyl alcohol) as a surfactant to improve oral bioavailability of the poorly soluble drug.

\section{MATERIALS AND METHODS}

\section{Materials}

Cilnidipine was received as a gift sample from Ravenbhel Healthcare Pvt. Ltd, Jammu, India. PLGA (75:25) 66,000-107,000 was bought from Sigma Aldrich, USA. PVA was supplied from Central Drug House (CDH) average molecular weight average 85,000-1, 24,000, New Delhi, India. Methanol and acetonitrile HPLC grade were supplied from Spectrochem Pvt. Ltd., Mumbai and HPLC grade water was supplied from Sd fine-Chem Ltd., Mumbai, India.

\section{Methods}

\section{Screening of excipient}

Screening of excipients was done on the basis of solubility of the drug [6]. Compatibility study of PLGA and cilnidipine was done by UV (Shimadzu UV1601, Tokyo, Japan), DSC (Pyris 6 DSC, Perkin Elmer, USA) and FTIR (Jasco FT/IR-410, Japan) to analyze any interaction between drug and the PLGA.

\section{Ultraviolet (UV) spectral analysis}

$20 \mu \mathrm{g} / \mathrm{ml}$ cilnidipine in methanol was scanned from $200 \mathrm{~nm}$ to 400 nm using UV spectrophotometer against methanol as blank. PLGA (75:25) was dissolved in ethyl acetate and scanned through UV region from 200-400 $\mathrm{nm}$. The drug-polymer mixture in methanol was also scanned through UV from $200-400 \mathrm{~nm}$.

\section{Fourier transforms infrared (FTIR) analysis}

FTIR spectrum for drug, polymer and drug-polymer mixture was recorded using potassium bromide pellet. Pellets were prepared with $1 \mathrm{mg}$ of the sample along with $200 \mathrm{mg}$ potassium bromide. FTIR spectrophotometer was operated from wavenumber 400 to $2000 \mathrm{~cm}^{-1}$ with a resolution of $4 \mathrm{~cm}^{-1}$. 


\section{Differential scanning calorimetry (DSC) analysis}

$1 \mathrm{mg}$ of sample (drug, polymer and drug-polymer mixture) was weighed in a standard open aluminium pan, while an empty pan of the same type was used as a reference. The heat cycle for a sample was set from 50 to $200{ }^{\circ} \mathrm{C}$ with the constant heating rate of $10^{\circ} \mathrm{C}$ per minute using nitrogen as an inert gas $(20 \mathrm{ml} / \mathrm{min})$. Calibration of temperature and heat flow was performed with indium.

\section{Preparation of polymeric nanoparticles of cilnidipine}

PLGA-cilnidipine (PLGA-CIL) nanoparticles were prepared by an emulsification solvent evaporation/diffusion method. The method is ideal for encapsulation of hydrophobic compounds like cilnidipine [7]. Briefly, the polymer PLGA was dissolved in ethyl acetate as per table 1 . $10 \mathrm{mg}$ of cilnidipine was dissolved in $1 \mathrm{ml}$ of methanol and then added to polymeric phase with intermittent vortexing using vortex mixer. The organic phase was then added to an aqueous phase of PVA to form solid in oil in water (S-O/W) emulsion. Once all the drug/polymer mixture was added to the PVA solution, the content was vortexed for $10 \mathrm{sec}$ at high speed. The resulting suspension was sonicated for 60 sec at $45 \%$ amplitude with a sonic disrupter. Immediately after sonication, the emulsion was poured into an excess of aqueous phase ( $0.1 \%$ PVA in water; $40 \mathrm{ml})$ for diffusion under rapid stirring on a magnetic stirrer. This colloidal suspension was kept on a magnetic stirrer for complete solvent evaporation for 5-6 h. The nanoparticles were then collected by centrifugation, washed 3 times with distilled Milli-Q treated water. Finally, they were re-suspended in $2 \mathrm{ml}$ of cryoprotectant solution (sucrose $2 \mathrm{w} / \mathrm{w}$ ) and trehalose $(5 \% \mathrm{w} / \mathrm{w})$ ), dried on a lyophilizer and stored at $4{ }^{\circ} \mathrm{C}$.

\section{HPLC method of analysis}

A reverse phase high-performance liquid chromatography (Shimadzu LC-10A VP, Shimadzu, Japan) method was modified and validated for the analysis of cilnidipine in different samples [8]. The mobile phase consisting of acetonitrile: water $(70: 30 \% \mathrm{v} / \mathrm{v})$ was used for the estimation of cilnidipine with UV detector set at $240 \mathrm{~nm}$. The run time for analysis was $10 \mathrm{~min}$ with a flow rate set at 1 $\mathrm{ml} / \mathrm{min}$. The injection volume used was $20 \mu \mathrm{L}$. The calibration curve of cilnidipine in rat plasma was also constructed in the range of 500 to $30000 \mathrm{ng} / \mathrm{ml}$ by plotting peak area vs. the corresponding drug concentration using the same HPLC conditions.

\section{Particle size distribution and polydispersity index (PDI)}

Particle size distribution and PDI were measured by Dynamic Light Scattering (DLS) technique using Zetasizer, (Malvern Zetasizer (NanoZS-90, UK) and analysed by "DTS nano" software. Droplet size of the prepared polymeric nanoparticle was determined by using photon correlation spectroscopy, which analyzes the fluctuations in light scattering due to Brownian movement of the particles. Light scattering was monitored at $25^{\circ} \mathrm{C}$ at $90^{\circ}$ angle. All the formulations were diluted nearly 200 times with double distilled water followed by vigorous shaking to get about 100-250 kilo counts per seconds for measurements. Average particle size (nm) and PDI were then recorded. The size analysis of a sample consisted of three measurements, and the results are expressed as the mean size \pm standard deviation (SD).

\section{Encapsulation efficiency and drug loading}

Lyophilized PLGA-CIL (5 mg) was dissolved in $2 \mathrm{ml}$ acetonitrile to extract cilnidipine into acetonitrile for determining the encapsulation efficiency. The samples in acetonitrile were gently shaken on a shaker for $1 \mathrm{~h}$ at room temperature to extract out cilnidipine from the nanoparticles into acetonitrile completely. These solutions were centrifuged at $10,000 \mathrm{rpm}$ and the supernatant was collected. Suspension $(20 \mu \mathrm{L})$ was dissolved in methanol $(1 \mathrm{ml})$ and was used for the estimation. Cilnidipine concentrations were measured spectrophotometrically at $\lambda_{\max } 240 \mathrm{~nm}$. A standard plot of cilnidipine $(0-10 \mu \mathrm{g} / \mathrm{ml})$ was prepared under identical conditions. The encapsulation efficiency (EE) of PLGA-CIL was calculated using the equation given below:

Encapsulation efficiency (\%)

$$
=\frac{\text { Total drug content in nanoparticles }}{\text { total drug amount }} \times 100
$$

The percent drug loading was calculated by dividing the total amount of drug extracted from the polymeric nanoparticles to the known weight of nanoparticles. The equation is given as

$$
\frac{\text { Drug content in nanoparticles }}{\text { Weight of nanoparticles }} \times 100
$$

\section{Transmission electron microscopy (TEM)}

Surface morphology of nanoparticle dispersion was observed using Morgagni 268D transmission electron microscope (TEM, FEI, USA) operated at $70 \mathrm{kV}$. The formulation was diluted with double distilled water nearly 100 times and sonicated for $5 \mathrm{~min}$. One drop of sonicated formulation was put on the copper grid coated with carbon film. Then the sample was negatively stained with $1 \%$ phosphotungstic acid (PTA) solution. The sample was dried in air before TEM observation.

\section{Scanning electron microscopy (SEM)}

SEM photomicrographs were taken by Carl Zeiss EVO 40, Germany for studying surface morphology. The sample was mounted onto stubs using double side adhesive carbon tape and then coated with gold before taking the images.

\section{Confocal laser scanning microscopy (CLSM)}

$5 \mathrm{~cm}$ long piece of the small intestine of the rat was taken and rinsed with Kreb's solution to remove all food residues. The formulation was prepared as per the method explained in the method of preparation with $0.03 \% \mathrm{w} / \mathrm{v}$ of Rodamine-B dye incorporated during the manufacturing process. $1 \mathrm{ml}$ of the formulation was placed in one sac and in another sac $1 \mathrm{ml}$ of the plain dye solution was kept and both the ends of the sac were ligated tightly and kept in Kreb's solution for $2 \mathrm{~h}$ with continuous stirring at $50 \mathrm{rpm}$ at $37^{\circ} \mathrm{C}$ with $95 \% \mathrm{O}_{2}$ maintained by an aerator. After $2 \mathrm{~h}$ intestinal sac was cut longitudinally and washed with Krebs solution to remove excess amount of dye. The small piece of the sac was mounted on the slide and observed under CLSM (Laser Confocal Microscope with Fluorescence Correlation SpectroscopyOlympus FluoView ${ }^{\mathrm{TM}}$ FV1000, Hamburg, Germany).

\section{Non-everted gut sac study}

The animal study was conducted according to protocol No.989 approved by Institutional Animal Ethics Committee (IAEC), Jamia Hamdard University, India. Male albino rats weighing 200-250 g were selected for experiments. The rats were housed in a clean cage with free access to food and water. Rats were fasted overnight and then sacrificed using diethyl ether just before the experiment. The intestine was surgically removed and flushed with saline. The small intestine was cut to obtain $5 \mathrm{~cm}$ long ileum. The suspension of the plain drug $(10 \mathrm{mg})$ was prepared using acacia and water. The drug suspension of $1 \mathrm{ml}$ was filled in the mucosal side and both the ends of sac were ligated tightly. The sac containing the drug suspension was immersed in a beaker containing $100 \mathrm{ml}$ of Krebs solution prewarmed at $37{ }^{\circ} \mathrm{C}$ by providing a continuous flow of heat to the beaker. Krebs solution was kept oxygenated with $95 \%$ oxygen for $120 \mathrm{~min}$ using an aerator. The transport of drug from mucosal to the serosal medium was measured by sampling from the serosal medium at a predetermined time interval and replenished with fresh Kreb's solution. The collected sample was filtered through 0.25 $\mu \mathrm{m}$ membrane filter and the amount of drug permeated through the gut sac was determined by UV spectrophotometer method. The experiment was repeated with formulation under same experimental conditions. The apparent permeability coefficient Papp of the plain drug and the formulation mentioned above was calculated from the following equation:

\section{$\mathrm{Papp}=\mathrm{F} / \mathrm{AxC}_{0} \mathrm{~cm}^{-1}$}

Where $\mathrm{F}$ is permeation flux ( $\mu \mathrm{g} / \mathrm{min}), \mathrm{A}$ is the surface area of the barrier membrane and $\mathrm{C}_{0}$ is the initial concentration of the drug in the mucosal medium.

Permeation flux (F) was calculated by taking the slope of the linear portion of the graph plotted between the cumulative amounts of drug permeated through sac against time ( $\mathrm{min}$ ). Surface area (A) was taken as $7.85 \mathrm{~cm}^{2}$ taking the length of sac $5 \mathrm{~cm}$ and assuming 
that it has a cylindrical shape with inner diameter measured as 0.50 $\mathrm{cm}$; taking no account of the microvilli and villi present in the mucosal side.

\section{In vitro drug release study}

In vitro release test was performed in $250 \mathrm{ml}$ of simulated gastric fluid of $\mathrm{pH} 1.2$ and simulated intestinal fluid of $\mathrm{pH} 6.8$ using USP XXIV method (Dissolution apparatus I. P.2, at $100 \mathrm{rpm}$ and $37 \pm 0.5$ ${ }^{\circ} \mathrm{C}$ ). A single dose of selected formulation (containing $10 \mathrm{mg}$ of cilnidipine) was placed in a treated dialysis bag and $1 \mathrm{ml}$ sample was withdrawn at a regular time interval $(0,15,30,60,120,240$ and 480 minute) and replaced with the same volume of buffer. The withdrawn $1 \mathrm{ml}$ sample was diluted to $3 \mathrm{ml}$ with mobile phase and analyzed for the drug content using developed RP-HPLC at $240 \mathrm{~nm}$. The in vitro release data was subjected to various kinetic models to analyse the release behaviour from a dosage form. The best fit model was determined on the basis of regression coefficient $\left(\mathrm{R}^{2}\right)$ value.

\section{Pharmacokinetic study}

The oral bioavailability study in albino Wistar rat was approved and performed in accordance with the guidelines of Institutional Animal Ethics Committee, Jamia Hamdard, New Delhi. The adult Wistar rats were obtained from the central animal house of Jamia Hamdard, New Delhi and kept under standard laboratory conditions at $25 \pm 2{ }^{\circ} \mathrm{C}$ temperature and $55 \pm 5 \%$ relative humidity, housed in polypropylene cages, with free access to a standard diet (Lipton feed, Mumbai, India) and water. The animal dose for conducting in vivo studies was calculated on the basis of body surface area ratio of a rat to that of a human being. The comparative in vivo pharmacokinetic study was carried out with 12 Wistar rats (weighing 200-250 g) which were divided randomly into three groups with four rats in each group. The study was conducted as a single dose design under fasting condition. For the present study, $1.02 \mathrm{mg} / \mathrm{kg}$ dose of cilnidipine calculated w. r. t humans was used. All the formulations were given orally using 18gauge oral feeding cannula according to the study plan. The rats were anaesthetized using diethyl ether, and $0.5 \mathrm{ml}$ blood sample was withdrawn from the tail vein of rat at 0 (pre-dose), $0.5,1,2,4,8,12$, 24 and collected in micro centrifuge tubes containing EDTA as an anticoagulant. The samples were then centrifuged at $5000 \mathrm{rpm}$ for $10 \mathrm{~min}$. The plasma was separated and stored at- $20^{\circ} \mathrm{C}$ until further analysis. To the collected plasma $1 \mathrm{ml}$ acetonitrile containing $1 \% \mathrm{v} / \mathrm{v}$ glacial acetic acid was added and the samples were vortexed for 2 min followed by centrifugation at $5000 \mathrm{rpm}$ for $10 \mathrm{~min}$. After that the supernatant was separated and reconstituted using $200 \mu \mathrm{l}$ mobile phase, it was analyzed by the RP-HPLC method as per mentioned procedure. The area value for the corresponding sample was observed, and the results were reported for triplicate observations. The area under the curve from $0 \rightarrow \mathrm{t}\left(\mathrm{AUC}_{0 \rightarrow 24}\right)$, from $0 \rightarrow \infty\left(\mathrm{AUC}_{0 \rightarrow \infty}\right)$, and area under first moment curve from $0 \rightarrow 24$ $\left(\right.$ AUMC $_{0 \rightarrow 24}$ ) were calculated with the help of Win Nonlin version 4.0 (Pharsight Corp., Mountain View, CA). A difference below the probability level of 0.05 was considered statistically significant. The pharmacokinetic data of different treatments were compared for statistical significance by a one-way ANOVA followed by TukeyKramer multiple comparisons test using Graph Pad Instat software (Graph Pad Software Inc., San Diego, CA).

\section{Pharmacodynamic study}

The antihypertensive activity of developed formulation was measured and compared with marketed and plain drug formulation by measuring the pressure of Albino rats by a Tail-cuff method using blood pressure (BP) measurement equipment (Le 5002 storage pressure meter). Hypertension was induced by injecting $20 \mathrm{mg}$ methylprednisolone/week through subcutaneous route to the trained rats. All the obtained results were expressed as mean \pm SD of 6 rats in each group.

\section{Stability study}

A stability study of the nanoparticle was carried out as per the guidelines given in the ICH Q1A (R2) (ICH topic Q1 (R2), 2009). Polymeric nanoparticle were stored under $25^{\circ} \mathrm{C} \pm 2{ }^{\circ} \mathrm{C} / 65 \% \pm 5 \% \mathrm{RH}$ and $40{ }^{\circ} \mathrm{C} \pm 2{ }^{\circ} \mathrm{C} / 75 \% \pm 5 \% \mathrm{RH}$ for a period of 3 mo in an oven (Thermo lab TH 90S). Samples at the predetermined time intervals
$(0,30,60$ and $90 \mathrm{~d})$ were withdrawn and analyzed for particle size distribution, precipitation, phase separation, re-dispensability and drug content. The amount of drug content remained after each interval was determined. A graph was plotted between $\log \%$ drug remaining vs. time (days). The degradation rate constant $(\mathrm{K})$ was determined from the following equation; the slope of the curve was determined from the graph.

$$
\text { Slope }=-\mathrm{K} / 2.303
$$

Where, $\mathrm{K}$ is the degradation rate constant.

The shelf life of the nanoparticle at $25{ }^{\circ} \mathrm{C}$ was calculated by calculating the time required to degrade $10 \%$ of the drug in the polymeric nanoparticle from the following equation.

$$
\mathrm{t}_{10 \%}=2.303 / \mathrm{K} \times \log (100 / 90)
$$

Where $t_{10 \%}$ is the time required to degrade $10 \%$ of the drug in the polymeric nanoparticles.

\section{RESULTS AND DISCUSSION}

\section{Excipient screening}

The optimized mixture of drug: and polymer was assessed for the compatibility using UV, DSC and FT-IR. UV spectrum of drug exhibited $\lambda_{\max }$ at $240 \mathrm{~nm}$ in methanol. Drug-polymer mixture taken in methanol did not alter the $\lambda_{\max }$ at $240 \mathrm{~nm}$ which corresponds to the identity of the drug [8]. DSC thermogram of the drug showed a melting endotherm at $109^{\circ} \mathrm{C}$ corresponding to drug's melting point. The endotherm was unaffected by the presence of polymer as shown in fig. 1. FTIR of the drug-polymer mixture showed the presence of drug as the peak related to the aromatic ring in cilnidipine was evident at $1697 \mathrm{~cm}^{-1}$ (fig. 2). In all cases, it is revealed that the drug was unaffected by the presence of PLGA.

\section{Formulation of polymeric nanoparticles}

Nanoparticles are prepared by various methods such as solvent evaporation, double emulsification method, nano-precipitation method, salting out and supercritical fluid technology [9-10]. In the present study, solvent evaporation method was employed. Solvent evaporation is suitable for drugs which exhibit some polarity in organic solvents such as methanol, chloroform or ethyl acetate. Solvent evaporation method assures small particle size for the nanoparticles than solvent precipitation method which is also a widely used method for preparation of nanoparticles [7, 9]. The polymer solution was prepared in ethyl acetate and then emulsified. Ethyl acetate was used as an organic phase as it belongs to generally recognised as safe (GRAS) category, with low toxicity (median lethal dose, LD50, in rats: $11.3 \mathrm{~g} / \mathrm{kg}$ ). Polyvinyl alcohol was used as a stabilizer for the prepared dispersed system as it prevents redispersibility problems. Variables such as the amount of polymer PLGA, PVA concentration and volume of ethyl acetate were studied for the responses such as the particle size, size distribution, percentage drug loading and encapsulation efficiency of any polymeric system [11]. In our study, a central composite design (CCD) was used to statistically optimise the formulation parameters and evaluate the main effects, interaction effects and quadratic effects of the formulation factors. The amount of PLGA tested was in the range 50-120 mg, PVA concentration was $1.0-3.0 \%$, and volume of ethyl acetate used was 2.0-5.0 ml. 15 runs including 8 factorial points, 6-star points and 1 centre point along with 5 replicated runs showing center points resulted in 20 experiments (table 1). The said design was chosen to estimate experimental errors and increase the precision. Each experimental run was repeated thrice.

\section{Influence on particle size}

Particle size is a critical factor for nanoparticle-based drug delivery system. It is one of the factors that control the kinetics of drug release. Generally, smaller particle size permits a faster release rate. The particle size values for the 20 trials showed a wide variation in response, i.e.; the response ranged from a minimum of $243 \mathrm{~nm}$ to a maximum of $661 \mathrm{~nm}$ (table 1). The data clearly indicated that the particle size value is strongly dependent on the selected variables. An increase in the mean particle size was observed when increasing concentration of PLGA was used for all the amount of PVA in the 
formulation (1-3\%). It was reported that an increase in the amount of PVA in the formulation may lead to the smaller particle size due to tight surface that is formed from PVA macromolecular chains at high concentration [12]

However, a high concentration of PVA is not suggested as it will hinder in vivo degradation. In addition, PVA has been found to have a carcinogenic potential and removal of excess PVA from the particle surface is difficult [13]. Our data supports that a lower concentration of PVA $(1 \% \mathrm{w} / \mathrm{v})$ is suitable to obtain well-controlled particle size formulations provided stability of dispersion is not compromised. Analysing the response surface of interactions of PLGA and ethyl acetate at constant PVA, we found that initially, with an increase of solvent volume, the particle size did not change much and then decreased with further increase in the volume of ethyl acetate (trial 13-20).

The volume of the organic phase is an important factor, as it controls the size of the nanoparticles. The particle size was also found to decrease with increase in the volume of the organic phase (trial 1, 5).
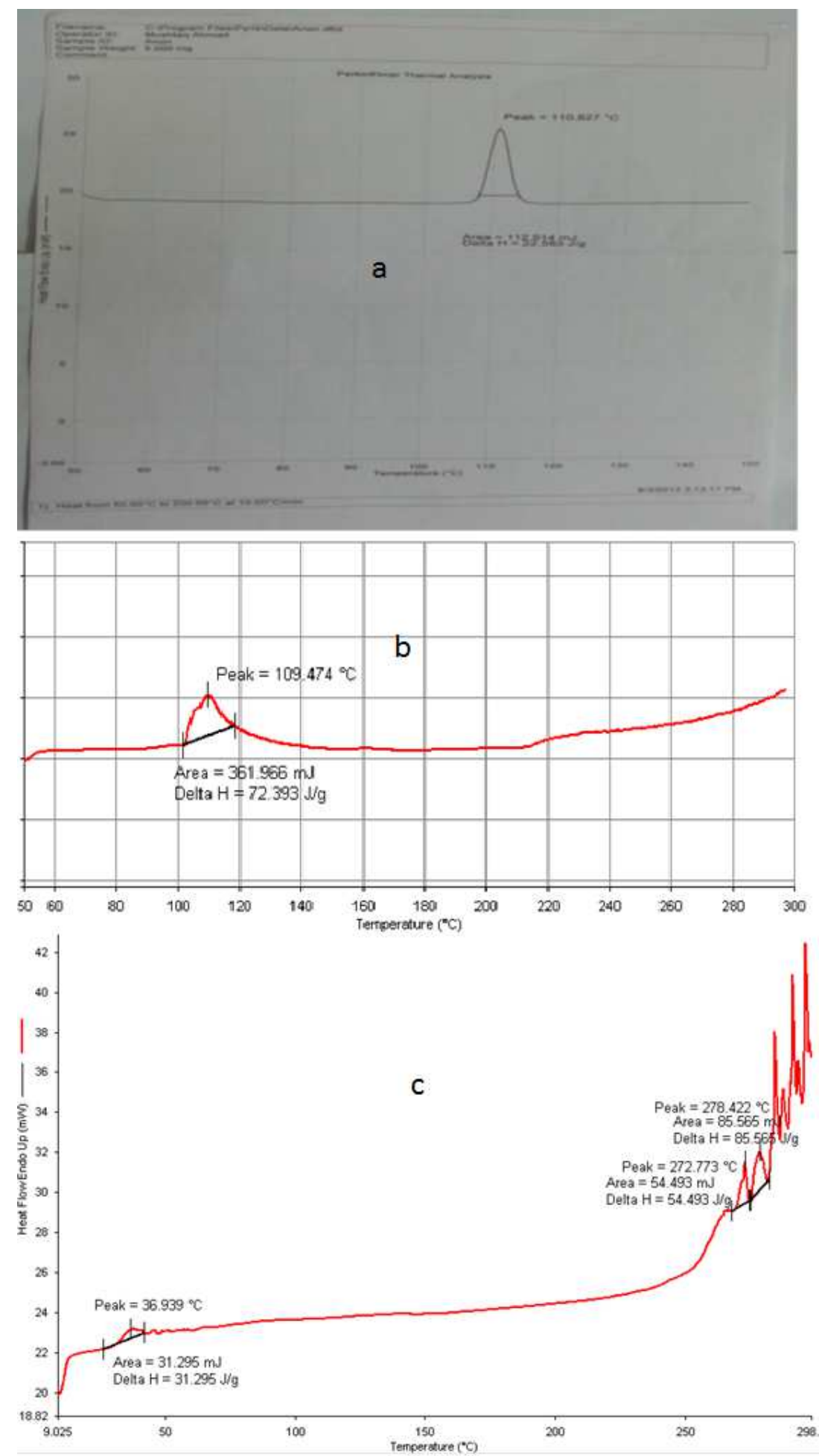

Fig. 1: DSC of (a) drug (b) drug: polymer (c) PLGA 


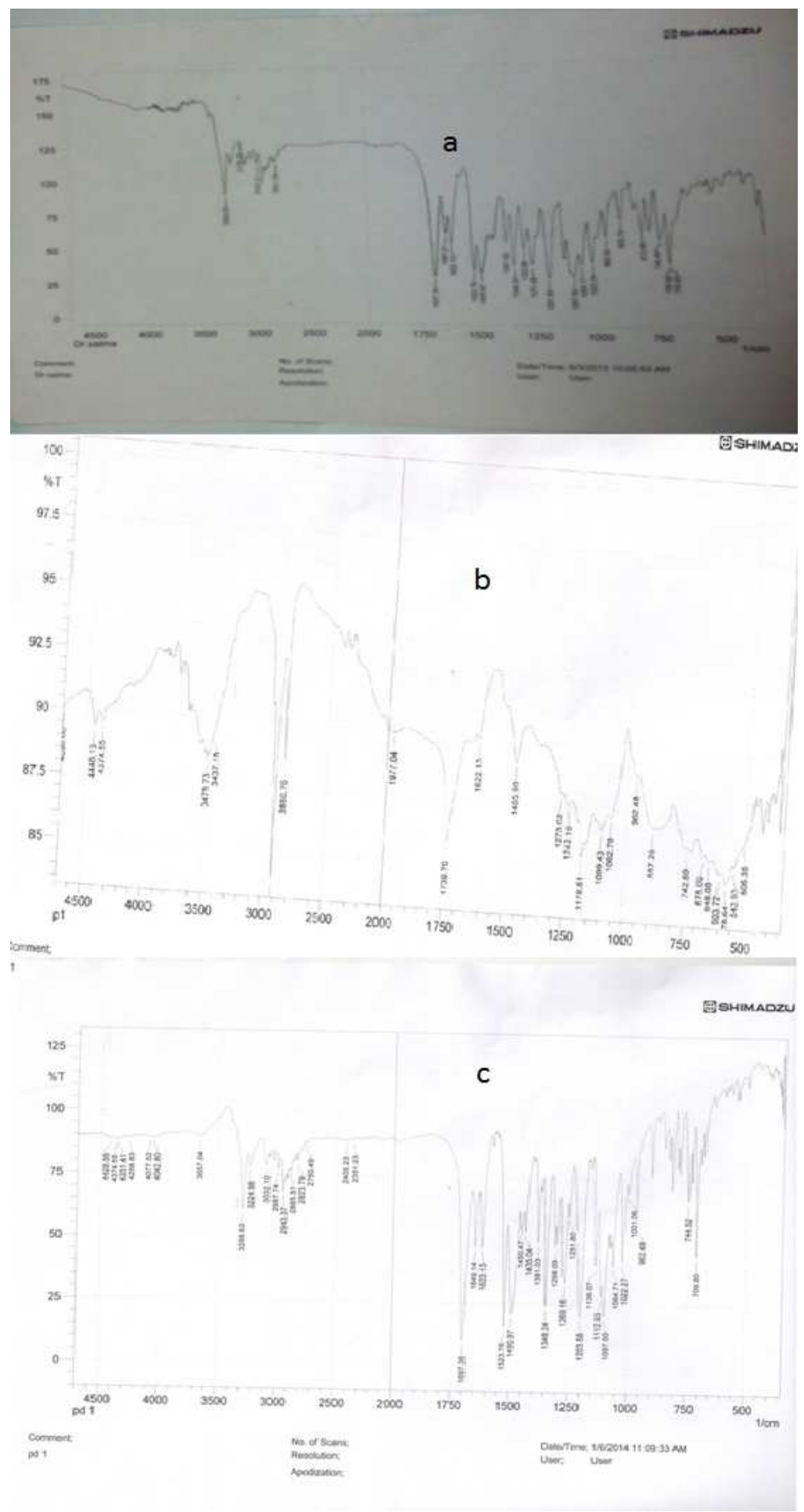

Fig. 2: FT-IR spectra of (a) cilnidipine (b) polymer (c) drug: polymer mixture

Usually, lower volume of organic phase rapidly mixes with the aqueous phase, causing faster precipitation and thus producing larger particles in comparison with the higher volume of the organic phase. Also, interfacial tension of ethyl acetate is low $(1.7 \mathrm{dyne} / \mathrm{cm})$, therefore it forms a stable primary emulsion which results in smallsized nanoparticles [14]. An increase in the polymer concentration led to an increase in the viscosity of the organic phase which resulted in the rapid diffusion of the organic phase into the aqueous phase. Coarse dispersions were obtained at higher polymer concentrations at constant PVA and organic solvent volume, possibly due to the insufficient amount of stabilizer present in the aqueous phase for that particular polymer amount, which led to larger particle size (trial 3,$4 ; 7,8$ ).

Dynamic light scattering showed particle size of $272 \mathrm{~nm}$ (fig. 3) which was higher than TEM analysis for the same optimised composition. This is because DLS measures the hydrodynamic diameter which is dependent on several factors such as the viscosity of the medium, diffusion coefficient, temperature, etc. than TEM which results due to scattering of electrons in sample irradiation. 

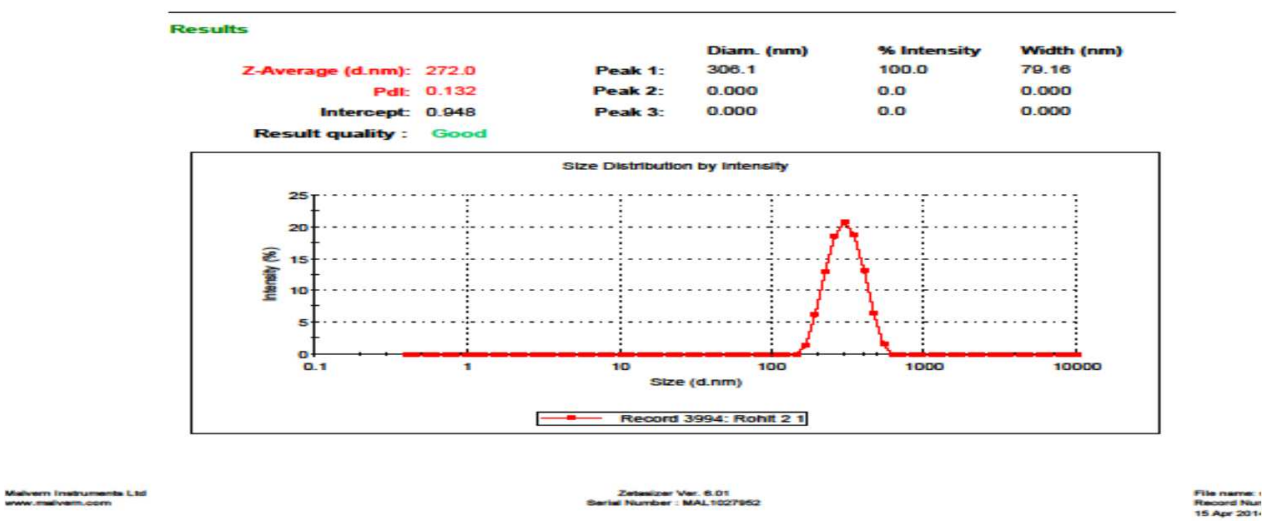

Fig. 3: Particle size distribution of polymeric nanoparticles of cilnidipine

\section{Influence on polydispersity index}

Generally, the polydispersity index is defined as the ratio of size deviation (or width of distribution) to mean particle diameter. The PDI value reflects the nanoparticle size distribution; samples with a wider range of particle sizes have higher PDI values (closer to 1), while samples consisting of evenly sized particles have lower PDI values (closer to 0 ). The polydispersity variations were found to be low in all the runs. A lower value indicated more monodisperse nanoparticles and greater particle stability, perhaps due to a higher volume of ethyl acetate used. Ethylacetate has low interfacial tension contributing to stable, evenly sized primary emulsion (table 1) [13]. In our study, we observed decrease in PDI with high concentration of PVA (trial 1, 7) which is also reported by Hylayqa [12] where high concentration of PVA showed decrease in PDI values for the same amount of PLGA, but the effect in our case is due to high volume of organic solvent used which assured the complete solubility of the polymer.

Table 1: Composition trials obtained from central composite design

\begin{tabular}{|c|c|c|c|c|c|c|c|}
\hline S. No. & PLGA (mg) & PVA (w/v) & Ethyl acetate (ml) & Particle size (nm) & PDI & Encapsulation efficiency (\%) & Drug loading (\%) \\
\hline 1. & 50 & 1 & 2 & 538.6 & 0.235 & 58 & 11 \\
\hline 2. & 120 & 1 & 2 & 475.2 & 0.240 & 56 & 9 \\
\hline 3. & 50 & 3 & 2 & 661.1 & 0.342 & 61 & 9 \\
\hline 4. & 120 & 3 & 2 & 624.1 & 0.310 & 64 & 10 \\
\hline 5. & 50 & 1 & 5 & 342.5 & 0.157 & 61 & 10 \\
\hline 6. & 120 & 1 & 5 & 283.6 & 0.144 & 86 & 7 \\
\hline 7. & 50 & 3 & 5 & 345.3 & 0.099 & 88 & 8 \\
\hline 8. & 120 & 3 & 5 & 515.3 & 0.328 & 84 & 9 \\
\hline 9. & 26.14 & 2 & 3.5 & 302.6 & 0.126 & 89 & 8 \\
\hline 10. & 143.86 & 2 & 3.5 & 292.2 & 0.185 & 91 & 6 \\
\hline 11. & 85 & 0.32 & 3.5 & 285.1 & 0.190 & 89 & 7 \\
\hline 12. & 85 & 3.68 & 3.5 & 272 & 0.132 & 81 & 11 \\
\hline 13. & 85 & 2 & 0.98 & 268 & 0.141 & 83 & 9 \\
\hline 14. & 85 & 2 & 6.02 & 255.3 & 0.136 & 88 & 9 \\
\hline 15. & 85 & 2 & 3.5 & 250.3 & 0.170 & 86 & 11 \\
\hline 16. & 85 & 2 & 3.5 & 243 & 0.140 & 88 & 6 \\
\hline 17. & 85 & 2 & 3.5 & 257 & 0.170 & 86 & 7 \\
\hline 18. & 85 & 2 & 3.5 & 252 & 0.150 & 89 & 8 \\
\hline 19. & 85 & 2 & 3.5 & 253 & 0.100 & 91 & 6 \\
\hline 20. & 85 & 2 & 3.5 & 252 & 0.130 & 89 & 7 \\
\hline
\end{tabular}

\section{Influence of preparation factors on encapsulation efficiency}

Encapsulation of drug in nanocarrier is an important index for drug delivery systems. In our study, encapsulation efficiency of polymeric nanoparticles (PLGA-cilnidipine; PLGA-CIL) reached up to $91 \%$ (table 1). High encapsulation efficiency is advantageous since it transports enough concentration at the target site and increases the residence time of the drug. The high encapsulation efficiency with PLGA can be attributed to several factors. First, the hydrophobic nature of PLGA makes it relatively easy to entrap hydrophobic cilnidipine into PLGA-CIL. Second, the hydrophobic nature of cilnidipine resulted in the minimum loss of the drug to the external aqueous phase during the formulation process. In our study, the encapsulation efficiency increased with increasing PLGA concentration at constant volume of ethyl acetate. With a higher volume of ethyl acetate, the entrapment of drug further increased perhaps the drug being hydrophobic in nature remained inside the polymer-organic phase which was stabilized by PVA (trial 6; trial 1520 ; trial 10). Also, the adopted method assured appropriate removal rate for ethyl acetate contributing to higher encapsulation [15].

\section{Influence on percentage drug loading}

Drug load in the drug delivery matrix plays a significant role on the rate and duration of drug release. Matrices with high drug loading possess a larger initial burst release than those with lower values [16]. In our study, the initial increase in polymer concentration increased the percentage drug loading at the same amount of PVA (trial 3, 4). Use of higher volume of solvent did not increase the drug loading (trial 7 and 8) (table1).

At higher PLGA concentration, initially there was no significant change observed for drug load with respect to PVA concentration, but drug loading increased with increase in PVA concentration (trial 4,6$)$.

\section{Transmission electron microscopy}

TEM is the most important technique for the study of microstructures because it directly produces 2D images at high resolution and it can capture any coexistent structures and microstructure transitions. In the present study, TEM image showed the smooth spherical surface of the polymeric nanoparticles with 
uniform size distribution. The result corroborated the particle size measurements through light scattering (fig. 4).

\section{Scanning electron microscopy}

To obtain more information about the particle size and shape, SEM analysis was also performed. The SEM photomicrograph (fig. 5) revealed that the lyophilized polymeric nanoparticles were spherical and in the nanometer range $(<200 \mathrm{~nm})$ than the polymer disc.

\section{Confocal laser scanning microscopy}

CLSM is one of the widely used image tools to determine the fate of drug delivery systems and biomolecules through the intestine. Through the use of fluorescence probes, it is possible to evaluate their behaviour, like: depth of penetration, cellular uptake, penetration routes into the intestine, interaction with the biological system and effect of the therapies on the intestine [17].

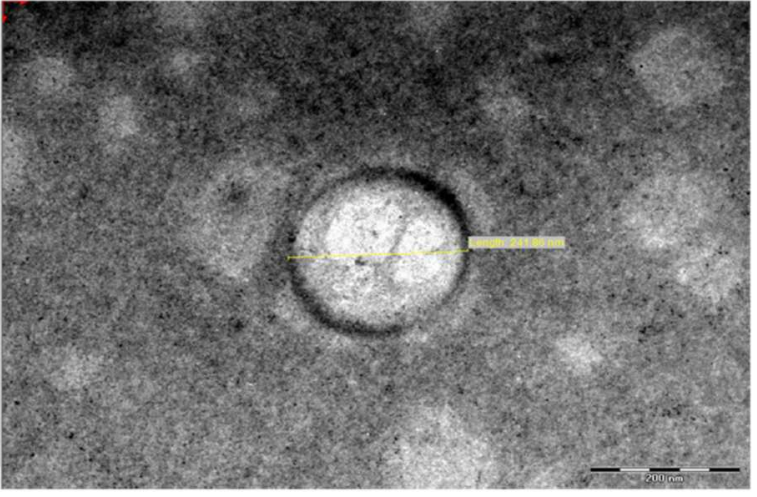

Fig. 4: TEM image of polymeric nanoparticles of cilnidipine

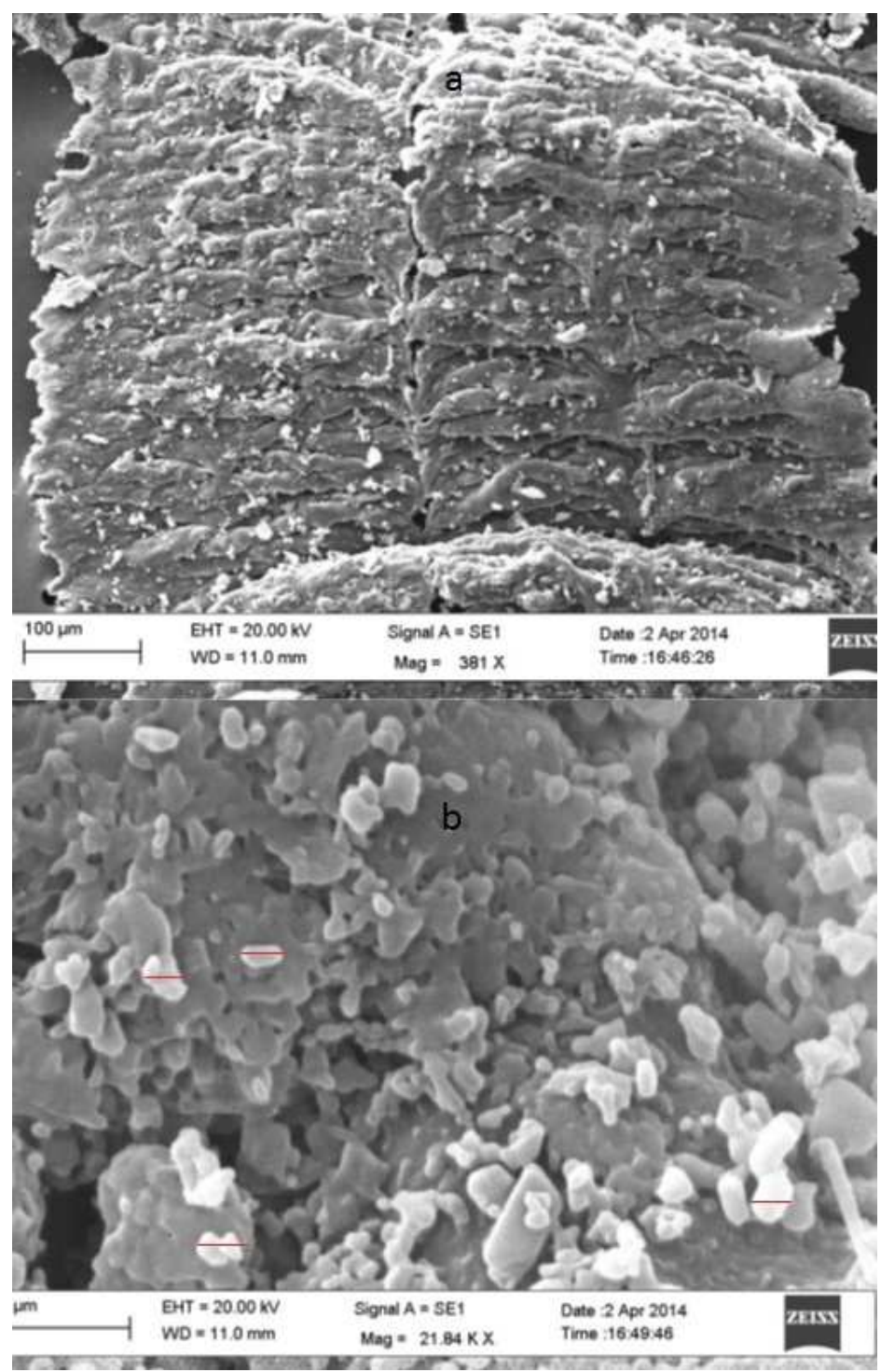

Fig. 5: SEM images of (a) polymer (b) polymeric nanoparticles

It generates high-resolution imaging, non-invasive optical sectioning and three-dimensional reconstructions in combination with sensitivity, selectivity and versatility of fluorescence measurement.

Rhodamine is a lipophilic dye. Because of its physicochemical properties, it is widely used to evaluate the dynamic properties of living cells such as membrane potential and ion concentration. It is also used to study the behaviour and the mechanism of permeation of lipid delivery systems like liposomes, solid lipid nanoparticles and nanostructured lipid carriers. It can be incorporated into the lipid bilayers promoting a marking and also mimicking lipophilic drugs since they both exhibit a similar partition coefficient (log P). The confocal laser scanning microscopy study clearly revealed that the dye incorporated into the formulation showed better penetration across 
the intestinal mucosa as compared to the plain dye solution probably due to the small size of the formulation and presence of PVA which is a nonionic mucoadhesive polymer assuring greater interpenetration [18]. The specific darkening of the intracellular spaces proves the point that the uptake of the dye incorporated into the formulation principally occurred by trans cellular transport. Fig. 6 clearly shows the better penetration of the dye that was incorporated in the formulation than the plain dye solution from the intestine.

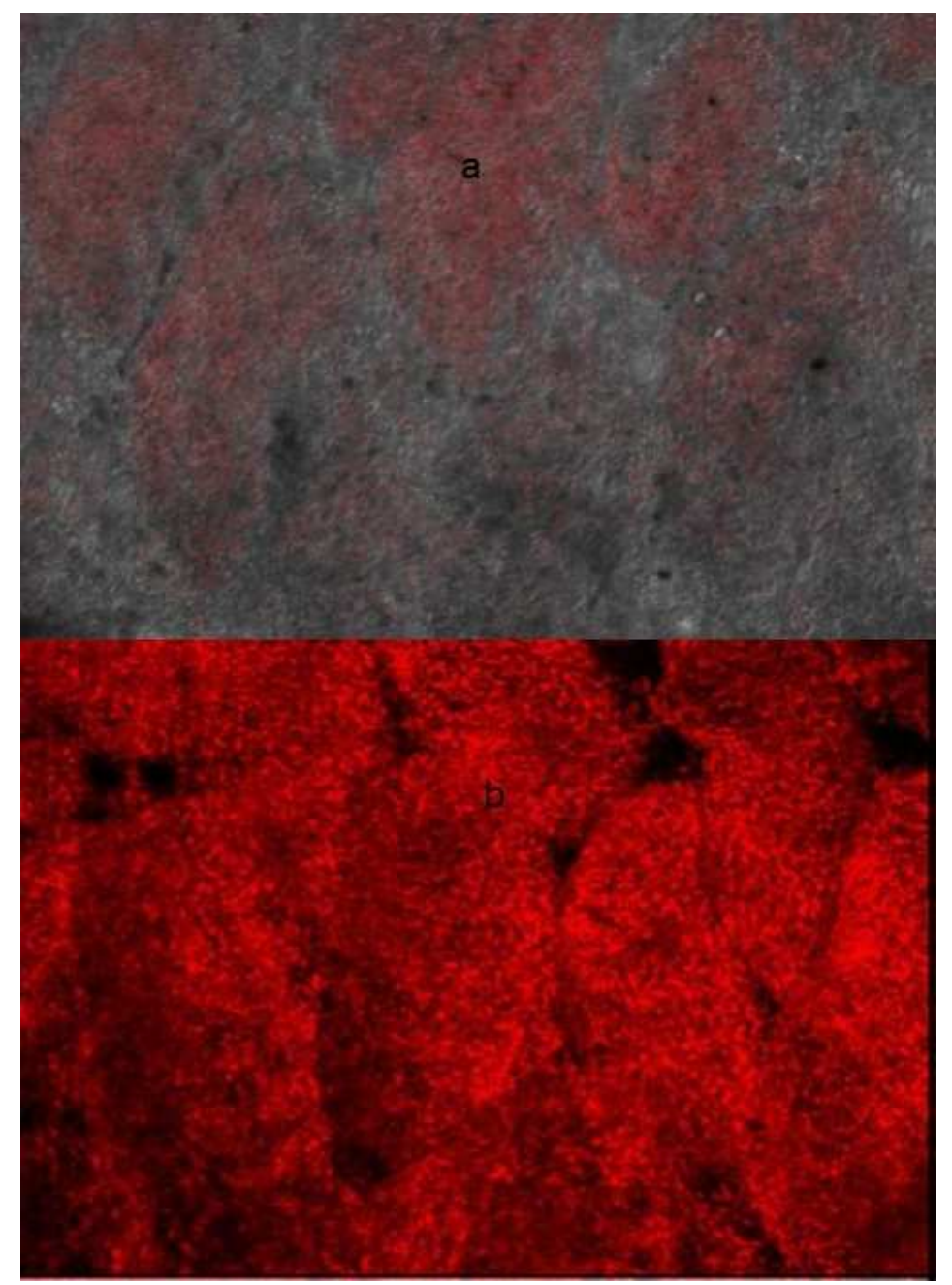

Fig. 6: CLSM image of (a) rodamiine solution (b) polymeric formulation with rodamine

\section{In vitro release}

The drug release from the nanoparticles is mostly dependent on the amount of drug loaded. The drug release is usually faster from the nanoparticles of lower drug entrapment efficiency [19]. The study is performed by using dialysis bag method [20].

In the acidic $\mathrm{pH} 1.2$, the dissolution study for $2 \mathrm{~h}$ showed only $11.5 \%$ cumulative drug release. But in the case of phosphate buffer pH6.8, in vitro release study of nanoparticles showed biphasic behaviour with initial burst release at first $2 \mathrm{~h}$ followed by sustained release pattern for $24 \mathrm{~h}$ [21]. Initial burst release effect was observed in the first 2 hour with a cumulative drug release of $45.3 \%$ due to sufficient drug loading. After $12 \mathrm{~h}$ cumulative \% drug release was measured as $81.57 \%$ indicating the release of entrapped drug (fig. 7). From the release profile, it can be seen that high initial drug burst was not observed as there was very little surface located drug in the nanoparticles and the majority of the drug was entrapped in the nanoparticles. This was corroborated by Hua et al. also for nimodipine loaded nanoparticles [22].

The high release was postulated to be because of small particle size of $272 \mathrm{~nm}$ assuring greater surface area. At the same time in vitro release study of the plain drug, suspension was also carried out under the same experimental conditions which showed only $44.66 \%$ cumulative $\%$ drug release.

The observations were analysed through different release models, and the formulation followed Korsmeyer-Peppas model (table 2) indicating diffusion-erosion controlled drug release system as $\mathrm{n}$ was between 0.5 and 1 .

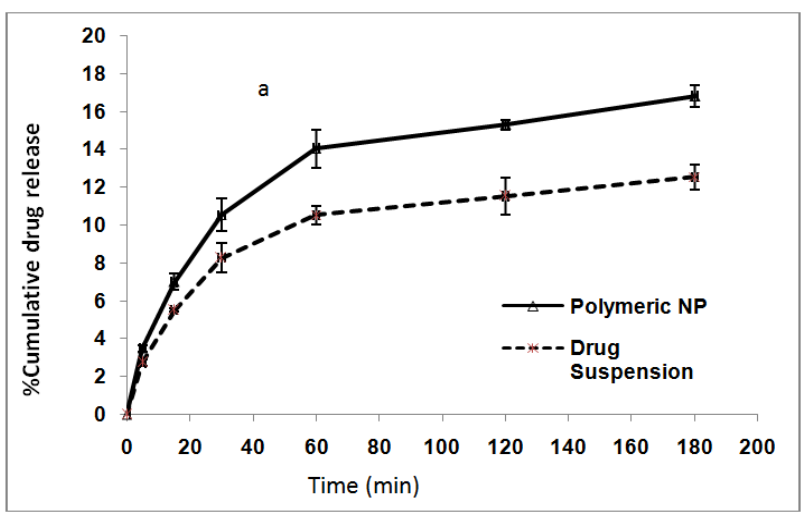




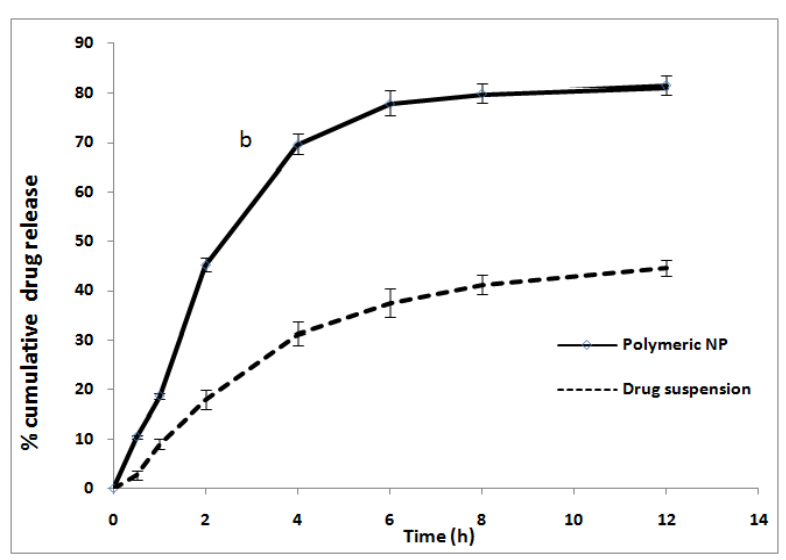

Fig. 7: In vitro release of drug in (a) buffer 1.2 (b) buffer 6.8 . Result is the mean \pm SD of three observations

\section{Ex-vivo gut permeation study}

Ruan et al. reported that there exists a good correlation between the permeability of drugs through non-everted rat intestine and their human absorption profile [23]. In our case, ex-vivo gut permeation study for cilnidipine nanoparticles showed that formulation composed of polymer and surfactant had better permeation compared to plain drug suspension which was performed at the same time under same experimental conditions. This is because Peyer's patches of ileum show high uptake for nanoparticles [24]. The apparent permeability coefficient $\left(\mathrm{P}_{\mathrm{app}}\right)$ for polymeric nanoparticle across ileum was found to be $0.013 \mathrm{~cm} / \mathrm{h}$, suggesting better absorption of nanoparticles than the drug suspension.

\section{Pharmacokinetic study}

Cilnidipine drug suspension, marketed cilnidipine tablet suspension and cilnidipine loaded PLGA nanoparticles were orally administered to male Wistar rats. The plasma concentration vs. time curve is shown in fig. 8. table 3 shows the pharmacokinetic parameters (peak concentration, time to reach peak concentration, the area under the curve 0-24 and area under the curve at infinity) of polymeric nanoparticles, drug suspension and tablet after oral administration. A remarkable increase in plasma concentration of cilnidipine was observed when nanoparticle group was studied than the control group. The $\mathrm{C}_{\max }$ of pure drug in suspension form was $1466.68 \mathrm{ng} / \mathrm{ml}$ at $4 \mathrm{~h}$ and for polymeric nanoparticles, it was found to be 4939.34 at $4 \mathrm{~h}$. These results imply a significant difference in bioavailability between nanoparticles and a drug suspension. AUC values for drug suspension and polymeric nanoparticles clearly demonstrate that the PLGA nanoparticles greatly improved the bioavailability for cilnidipine. The marketed tablet formulation though showed a slight increase in $\mathrm{C}_{\max }$ but was less than nanoparticles. The improved bioavailability is perhaps due nano-scale particles which are easily absorbed into the folds of the intestinal wall and larger particle surface area to volume leading to rapid drug dissolution [25].

Table 2: Release kinetic study for the optimised polymeric nanoparticle of cilnidipine

\begin{tabular}{ll}
\hline Kinetic modeling & $\mathbf{R}^{\mathbf{2}}$ Value \\
\hline Zero Order Kinetics (slope of percent drug release Vs time) & 0.737 \\
First Order kinetics (slope of log percent drug release Vs time) & 0.820 \\
Higuchi Model (slope of percent cumulative drug release Vs square root of time) & 0.877 \\
Haxon-Crowell model (slope of cube root of percent cumulative drug release Vs time) & 0.794 \\
Korsmeyer-Peppas Model (slope of log percent drug release Vs Log time) & 0.926 \\
\hline
\end{tabular}

PLGA-nanoparticles showed longer half-life of $17 \mathrm{~h}$ than marketed formulation with $t_{1 / 2}$ of $16 \mathrm{~h}$. This is perhaps due to prolonged absorption and sustained release of drug from polymeric carrier. In all, the bioavailability of nanoparticles was observed to be nearly three-fold higher than the tablet suspension given orally.

Entrapment of drug in biodegradable nanoparticles resulted in reduced apparent clearance, longer apparent circulation half-life and lower apparent volume of distribution, leading to long-lasting drug concentration in the plasma (table 3).

It is postulated that encapsulation of drug molecules in nanoparticles significantly reduces the apparent drug clearance from plasma, thereby enhancing the apparent drug circulation half-life and potential cumulative drug delivery to the target tissues [26]. It must have also protected the drug from degradation by adverse conditions in the gastrointestinal tract [27].

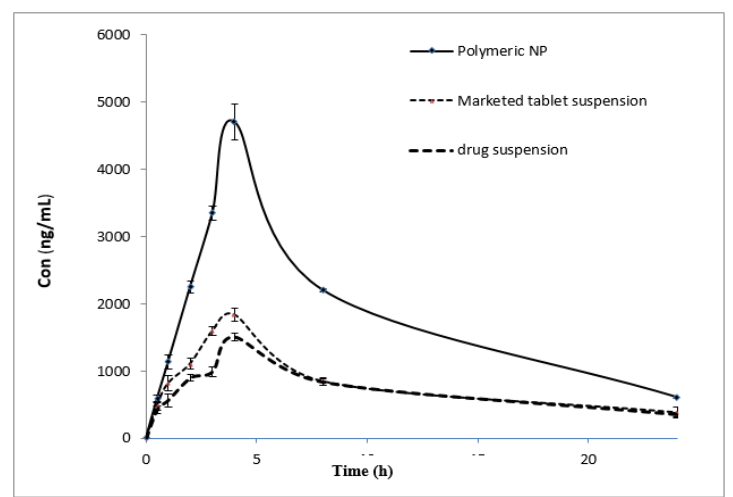

Fig. 8: In vivo absorption profile of cilnidipine using different formulations. Result is the mean \pm SD of three observations at each time point

Table 3: Pharmacokinetic parameters for polymeric nanoparticles

\begin{tabular}{|c|c|c|c|c|c|c|c|}
\hline Formulation & $\begin{array}{l}t_{\max }{ }^{a} \\
\text { (h) }\end{array}$ & $\begin{array}{l}C_{\max }{ }^{b} \\
(\mathrm{ng} / \mathrm{ml})\end{array}$ & $\begin{array}{l}\mathrm{AUC}_{0 \rightarrow \mathrm{t}^{\mathrm{c}}} \\
(\mathrm{ng} / \mathrm{ml})\end{array}$ & $\begin{array}{l}\text { AUC }_{0 \rightarrow \infty} \\
(\mathbf{n g} / \mathrm{ml})\end{array}$ & $\begin{array}{l}\text { AUMC }_{0-\mathrm{t}} \\
\text { (ng/ml) }\end{array}$ & $\begin{array}{l}\text { AUMC }_{0-\infty} \\
(\mathrm{ng} / \mathrm{ml})\end{array}$ & Vd (Litres) \\
\hline Polymeric nanoparticles (PNP) & 4 & 4939.34 & 45798.96 & 60371.42 & 352681 & 1063571 & 0.176 \\
\hline Tablet suspension & 4 & 1868.11 & 19576.96 & 27555.58 & 160933.3 & 539408.7 & 0.195 \\
\hline API drug suspension & 4 & 1466.68 & 16541.21 & 25590.11 & 141907.1 & 631134.4 & 0.246 \\
\hline
\end{tabular}

atime of peak concentration, bpeak of maximum concentration; ${ }^{c}$ area under the concentration-time profile, curve until last observation.

\section{Pharmacodynamic study}

Glucocorticoids, like methylprednisolone, are known to cause systemic hypertension on subcutaneous administration [28]. In our study, hypertension was successfully induced in all groups except control by methylprednisolone acetate administration at a dose 20 $\mathrm{mg} / \mathrm{kg}$ body weight to rats. A significant rise of $44 \mathrm{mmHg}$ was obtained in systolic blood pressure (SBP) during the one-week 
treatment with methylprednisolone $(160 \pm 11.13)$ as compared to control $(116.333 \pm 2.516)$. Fig. 9 shows that cilnidipine treatment reduced the pressure significantly in the MP+polymeric nanoparticle, MP+Neual-10 Tablet and MP+Drug Suspension treated groups on day one of the treatment, however, the effect was sustained well by polymeric nanoparticles with once a day administration for consecutive two days.

A significant rise of $11 \mathrm{mmHg}$ was obtained in diastolic blood pressure (DBP) during the one-week treatment with MP $(99.67 \pm 1.43 \mathrm{mmHg})$ as compared to control $(88.67 \pm 2.52 \mathrm{mmHg}$ ) (fig. 9). Cilnidipine treatment reduced the diastolic blood pressure significantly in MP+polymeric nanoparticles $(93 \pm 2.0)$. There was no change in heart rate after the administration of cilnidipine polymeric nanoparticles.
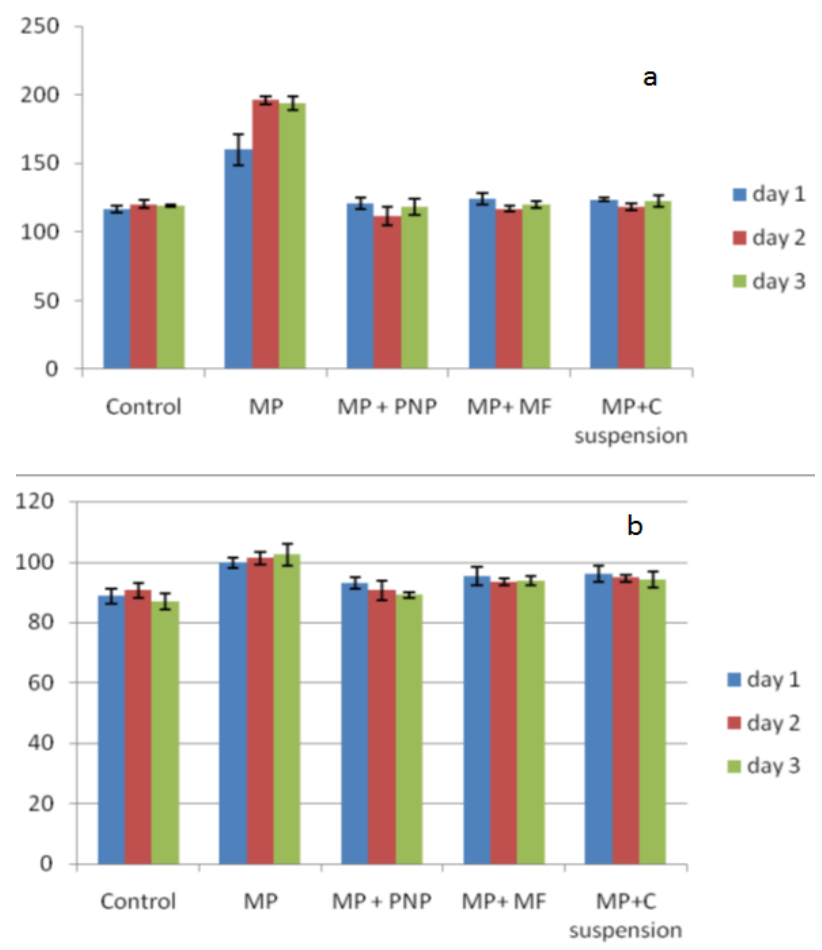

Fig. 9: Blood pressure of Wistar rats (mmHg) a-(Systolic) and b(Diastolic) (Results are the mean $\pm S D$ of three observations at each time point)

\section{CONCLUSION}

PLGA nanoparticles of cilnidipine were prepared by emulsificationsolvent evaporation method. The particles had a spherical shape and particle size was in the range of 242-661 nm. The polymeric nanoparticles showed high encapsulation for the drug leading to controlled release for $12 \mathrm{~h}$ in simulated intestinal fluid. Important variables such as PLGA concentration, PVA concentration and volume of solvent were observed to influence the performance of nanoparticles. The optimized cilnidipine nanoparticles showed greater permeation across non-everted rat intestine which was further validated through in vivo pharmacokinetic study, where nearly two-fold increase in oral bioavailability was observed than marketed tablet-Neual 10.

\section{ACKNOWLEDGMENT}

We wish to thank the Department of Pharmaceutics, Jamia Hamdard, New Delhi for providing the instrumentation facilities.

\section{CONFLICT OF INTERESTS}

The authors declare that they have no conflict of interest.

\section{REFERENCES}

1. Tandel H, Raval K, Nayani A, Upadhay M. Preparation and evaluation of cilnidipine microemulsion. J Pharm Bioallied Sci 2012;4 Suppl 1:114-5.
2. Prajapati ST, Maheshwari PD, Patel CN. Formulation and evaluation of orodispersible tablets of cilnidipine by spray drying technique. World J Pharm Sci 2015;4:1526-39.

3. des Rieux A, Fievez V, Garinot M, Schneider YJ, Préat V. Nanoparticles as potential oral delivery systems of proteins and vaccines: a mechanistic approach. J Controlled Release 2006;116:1-27.

4. Semete B, Booysen L, Lemmer Y, Kalombo L, Katata L, Verschoor J, Swai HS. In vivo evaluation of the biodistribution and safety of PLGA nanoparticles as drug delivery systems. Nanomedicine 2010;6:662-71.

5. Jawahar N, Venkatesh DN, Sureshkumar R, Senthil V, Ganesh GNK, Vinoth P, et al. Development and characterization of PLGA-nanoparticles containing carvedilol. J Pharm Sci Res 2009;1:123-8.

6. Kim TH, Jeong YI, Jin SG, Pei J, Jung TY, Kim IY, et al. Preparation of polylactide-co-glycolide nanoparticles incorporating celecoxib and their antitumor activity against brain tumor cells. Int J Nanomed 2011;6:2621-31.

7. Tomoda K, Terashima H, Suzuki K, Inagi T, Terada H, Makino K. Enhanced transdermal delivery of indomethacin-loaded PLGA nanoparticles by iontophoresis. Coll Surf B 2011;88:706-10.

8. Kokilambigai KS, Lakshmi KS. Analytical methodologies for determination of cilnidipine: an overview. Int J Pharm Pharm Sci 2014;6:36-8.

9. Geckeler KE, Rao JP. Polymer nanoparticles preparation techniques and size control parameters. Prog Polym Sci 2011;36:887-913.

10. Alshamsan A. Nanoprecipitation is more efficient than emulsion solvent evaporation method to encapsulate cucurbitacin I in PLGA nanoparticles. Saudi Pharm J 2014;22;219-22.

11. Li Z, Tao W, Zhang D, Wu C, Song B, Wang S, et al. The studies of PLGA nanoparticles loading atorvastatin calcium for oral administration in vitro and in vivo. Asian J Pharm Sci 2016. Doi:10.1016/j.ajps.2016.08.006.

12. Halayqa M, Domanska U. PLGA biodegradable nanoparticles containing perphenazine or chlorpromazine hydrochloride: effect of formulation and release. Int $\mathrm{J}$ Mol Sci 2014;15:23909-23.

13. DeMerlis CC, Schoneker DR. Review of the oral toxicity of polyvinyl alcohol (PVA). Food Chem Toxicol 2003;41:319-26.

14. Vineetha P, Vadaparthia PRR, Kumara K, Dileep B, Babu J, Veerabhadra Rao A, et al. Influence of organic solvents on nanoparticle formation and surfactants on release behaviour in vitro using costunolide as a model anticancer agent. Int J Pharm Pharm Sci 2014;6:638-45.

15. Zhang X, Yuan Y, Shan X, Sheng Y, Xu F, Liu C. Effects of surfactant and solvent on the encapsulation efficiency and size in using double emulsion method for preparing bovine hemoglobin loaded nanoparticles as blood substitutes. J Biomed Eng 2009;26:116-21.

16. Makadia HK, Siegel SJ. Poly lactic-co-glycolic acid as biodegradable controlled drug delivery carrier. Polymers 2011;3:1377-97.

17. Rossetti FC, Depieri LV, Bentley MVLB. Confocal laser scanning microscopy as a tool for the investigation of skin drug delivery systems and diagnosis; 2013. Available from: http: cdn.intechopen.com/pdfs-wm/43810. [Last accessed on 15 Sep 2016].

18. Karolewicz B. A review of polymers as multifunctional excipients in drug dosage form technology. Saudi Pharm J 2016;24:5525-36.

19. Gandhi A, Jana S, Kumar K. In vitro release of acyclovir-loaded Eudragit RLPO @nanoparticles for sustained drug delivery. Int J Biol Macromol 2014;67:478-82.

20. Ahmad J, Mir SR, Kohli K, Chuttani K, Mishra AK, Panda AK, et al. Solid nanoemulsion preconcentrate for oral delivery of paclitaxel: formulation, design, biodistribution and scintigraphy imaging. Biomed Res Int 2014;14:1-12.

21. Seju U, Kumar A, Sawant KK. Development and evaluation of olanzapine-loaded PLGA nanoparticles for nose-to-brain 
delivery: In vitro and in vivo studies. Acta Biomaterial 2011;7:4169-76.

22. Hua Y, Jianga X, Ding $\mathrm{Y}$, Zhang L, Yang $\mathrm{C}$, Zhang J, et al. Preparation and drug release behaviours of nimodipine-loaded poly(caprolactone)-poly(ethylene oxide)-polylactide amphiphilic copolymer nanoparticle. Biomaterials 2003;24: 2395-404.

23. Ruan LP, Chen S, Yu BY, Zhu DN, Cordell GA, Qiu SX. Prediction of human absorption of natural compounds by the non-everted rat intestinal sac model. Eur J Med Chem 2006;41:605-10.

24. Zakeri-Milani P, Delf Loveymi B, Jelvehgari M, Valizadeh H. The characteristics and improved intestinal permeability of vancomycin PLGA-nanoparticles as colloidal drug delivery system. Colloids Surf B 2013;103:174-81.

25. MaY, Zhao X, Li J, Shen Q. The comparison of different daidzeinPLGA nanoparticles in increasing its oral bioavailability. Int J Nanomed 2012;7:559-70.
26. Kadam RS, Bourne DWA, Kompella UB. Nano-advantage in enhanced drug delivery with biodegradable nanoparticles: contribution of reduced clearance. Drug Metab Dispos 2012;40:1380-8.

27. Mittal G, Kumar MN. The impact of polymeric nanoparticles on oral pharmacokinetics: a dose-dependent case study with estradiol. J Pharm Sci 2009;98:3730-4.

28. Ahmad J, Kohli K, Amin S. Formulation of self-nano emulsifying drug delivery system for telmisartan with improved dissolution and oral bioavailability. J Dispersion Sci Technol 2011;32:958-68.

\section{How to cite this article}

- Rohit Mishra, Showkat R Mir, Saima Amin. Polymeric nanoparticles for improved bioavailability of cilnidipine. Int J Pharm Pharm Sci 2017;9(4):129-139. 